Research Article

\title{
Impact Characteristics and Fatigue Life Analysis of Multi-Wire Recoil Spring for Guns
}

\author{
Zhifang Wei $\mathbb{D},{ }^{1}$ Xiaolian Zhang $\mathbb{D},^{2}$ Yecang $\mathrm{Hu} \mathbb{D}^{\mathrm{D}},{ }^{3}$ and Yangyang Cheng $\mathbb{D}^{1}$ \\ ${ }^{1}$ College of Mechatronics Engineering, North University of China, No. 3, Xueyuan Road, Jiancaoping, Taiyuan, Shanxi, China \\ ${ }^{2}$ Southwest Technology and Engineering Research Institute, No. 33, Yuzhou Road, Shiqiaopu, Jiulongpo, Chongqing, China \\ ${ }^{3}$ Sichuan Academy of Aerospace Technology, No. 118, North Aerospace Road, Longquanyi, Chengdu, Sichuan, China
}

Correspondence should be addressed to Zhifang Wei; wzhifang@nuc.edu.cn

Received 1 August 2020; Revised 10 September 2020; Accepted 24 October 2020; Published 16 November 2020

Academic Editor: Moon Gu Lee

Copyright ( 92020 Zhifang Wei et al. This is an open access article distributed under the Creative Commons Attribution License, which permits unrestricted use, distribution, and reproduction in any medium, provided the original work is properly cited.

Recoil spring is a key part in automatic or semi-automatic weapons re-entry mechanism. Because the stranded wire helical spring (SWHS) has longer fatigue life than an ordinary single-wire cylindrically helical spring, it is often used as a recoil spring in various weapons. Due to the lack of in-depth research on the dynamic characteristics of the current multi-wire recoil spring in recoil and re-entry processes, the fatigue life analysis of the current multi-wire recoil spring usually only considers uniform loading and does not consider dynamic impact loads, which cannot meet modern design requirements. Therefore, this paper proposes a research method for fatigue life prediction analysis of multi-wire recoil spring. Firstly, based on the secondary development of UG, a threewire recoil spring parameterized model for a gun is established. Secondly, ABAQUS is used to carry out a finite element analysis of its dynamic response characteristics under impact, and experimental verification is performed. Then, based on the stress-time history curve of the dangerous position obtained by finite element analysis, the rain flow counting method is used to obtain the fatigue stress spectrum of recoil spring. Finally, according to the Miner fatigue cumulative damage theory, the fatigue life prediction of the recoil spring based on the $\mathrm{S}-\mathrm{N}$ curve of the material is compared with experimental results. The research results show that the recoil spring has obvious transient characteristics during the impact of the bolt carrier. The impact velocity is far greater than the propagation speed of the stress wave in the recoil spring, which easily causes the spring coils to squeeze each other. The maximum stress occurs at the fixed end of the spring. And the mean fatigue curve (50\% survival rate) is used to predict the life of the recoil spring. The calculation result is $8.6 \%$ different from the experiment value, which proves that the method has certain reliability.

\section{Introduction}

Recoil spring is a key part used in the re-entry mechanism of automatic or semi-automatic weapons to store energy during the recoil of the moving parts and release energy during the re-entry process to make the moving parts complete the reentry. During the recoil and re-entry process, the moving parts drive the corresponding mechanisms to complete automatic actions, such as shell withdrawal, bomb feeding, locking, and firing. Because the SWHS has longer fatigue life than an ordinary single-wire cylindrically helical spring, it is often used as a recoil spring in various weapons. Recoil spring mainly bears high-speed impact loads during reciprocating motion, which causes the spring to deform quickly; then the dense waves are formed between the coils, which causes chattering, resulting in uneven deformation and stress distribution, making a part of the spring where the stress is concentrated to form alternating tension and compression stress. With the number of alternating changes and the increase in stress amplitude, the free length of the spring will decrease, the spring force will weaken, and sometimes even fatigue cracks will occur. Fatigue failure of recoil spring will lead to the weakness of the automaton, resulting in the recoil and re-entry not in place, affecting the shooting accuracy. Therefore, the analysis of impact characteristics and fatigue life estimation of recoil spring is very important.

The multi-wire recoil spring is a SWHS and it is a unique cylindrically helical spring, which is reeled by a strand that is 
formed of 2 16 steel wires. Each steel wire is a secondary helix form in space, so the structure is relatively complicated. Due to the complex contact friction between the wires, the multi-wire recoil spring has nonlinear characteristics of stiffness and damping. Therefore, when the multi-wire recoil spring is subjected to high-speed impact loads during recoil and re-entry processes, its impact characteristics are different from ordinary single-wire cylindrically helical springs.

Peng et al. [1] proposed a parametric modeling method and the corresponding 3D model of a closed-end stranded wire helical spring based on the forming principle of the spring and performed numerical simulation to test the validity of the parametric modeling method through Pro/ Engineering. Phillips and Costello [2] proposed a theory to analyze the large static axial response of stranded wire springs, and the study found that the response of a typical tension or compression spring was weakly nonlinear at large spring strain and relatively insensitive to the type of end condition. Costello and Phillips [3] considered the large static deflection of an axially loaded helical spring and further analyzed the axial response of stranded wire helical springs based on theory. It was found that the axial stiffness of the stranded wire helical spring has a good engineering approximation with the same number of independently acting untwisted helical wires, and the theoretical analysis was verified by experiments. Lee [4] used the pseudospectral method to carry out free vibration analysis of cylindrical helical springs. The numerical calculation results were in good agreement with the results obtained by the transfer matrix method and the dynamic stiffness method. Yu [5] analyzed the dynamic stress of single-wire recoil spring using graphical method and stress wave principle and pointed out that when recoil spring is impacted, the maximum stress exceeds the static stress generated when the coils contact. Min et al. [6] analyzed the dynamic characteristics of SWHS based on theory and gave calculation examples of SWHS vibration displacement. Wang et al. [7] used the finite element method to study the motion forms of the spring particles when the closed-end SWHS was impacted. It was concluded that if the impact speed is too large, the spring coils will compress and merge. Further, Wang et al. [8] carried out experimental research on the impact load characteristics of a SWHS and pointed out that when a SWHS is subjected to impact loads, the displacement and velocity no longer have an axial linear distribution. Although the above scholars have conducted research on the impact characteristics of SWHS, the load conditions experienced by SWHS in the above studies do not exactly match the actual working conditions of the multi-wire recoil spring. Therefore, the research on the dynamic characteristics of the multi-wire recoil spring of automatic weapons during recoil and re-entry is not specific.

Čakmak et al. [9] numerically modeled and analyzed helical spring fatigue and proposed novel helical spring stress and deflection correction factors based on the theory of elasticity and finite element analysis. Zhang [10] introduced a method based on eternal fatigue life combined with $\mathrm{S}-\mathrm{N}$ curve to analyze the fatigue life of spring. Yu et al. [11] based on the stress-strength interference model to carry out the reliability design of the fatigue strength of the cylindrically helical spring. Lei [12] performed a finite element analysis of twisted fretting wear of SWHS and provided a reference for the in-depth understanding of the mechanism of twisted fretting wear of the spring. Fatigue life research on SWHS is rare at present, and multi-wire recoil springs of automatic weapons are subject to high-speed impact during launch. Therefore, it is of great significance to apply the results of multi-wire recoil spring impact characteristics analysis to its fatigue life analysis.

In this paper, a three-wire SWHS used as a recoil spring for a caliber machine gun is taken as the research object. A multi-wire recoil spring geometric model parametric modeling method is proposed using continuous multisegment functions, sweep modeling technology, and UG secondary development. Based on the kinematic characteristics of the bolt carrier, the actual operating conditions of the multi-wire recoil spring are analyzed, and the finite element modeling of the impact response characteristics of the multi-wire recoil spring under actual working conditions is studied. In addition, based on the actual operating conditions, a set of test devices for the impact characteristics of the recoil spring is developed, and the dynamic parameters such as the motion displacement, velocity, acceleration, stress, and strain of the various points of the spring coils are analyzed. And an effective fatigue stress spectrum is obtained by analyzing the dangerous points in time domain and applying the rain flow counting method. Then, based on the results of dynamic characteristics analysis, the S-N curve of recoil spring material (55Si2Mn) is modified to obtain the recoil spring fatigue life curve. Finally, the engineering calculation based on Miner theory is used to predict the fatigue life of recoil spring. It can provide some theoretical support for the research of other springs or automatic weapon recoil spring.

\section{Multi-Wire Recoil Spring Parametric Modeling}

Due to the application of a large number of secondary helix geometric features, the modeling of multi-wire recoil spring is a bit complicated and time-consuming and laborious, and because the modeling is not standardized, it often leads to errors in the design parameters, making interference between wire and wire in the geometric model. Therefore, the standardization and parameterization of multi-wire recoil spring 3D modeling are necessary for the accurate design of multi-wire recoil spring.

\subsection{The Center Curve Equations and Geometric Parameters} Calculation of Multi-Wire Recoil Spring. Figure 1 shows the geometric model of the three-wire recoil spring for a machine gun studied in this paper. In the figure, $D$ is the middle diameter of the recoil spring. $D_{2}$ is the outer diameter of the recoil spring. $H_{0}$ is the free height of the recoil spring. $d c$ is the diameter of the strand. $t c$ is the pitch of the strand. $\beta$ is the twist angle of strand. $d$ is the diameter of the wire. $d_{2}$ is the diameter of the circle formed by the center of the wires. 

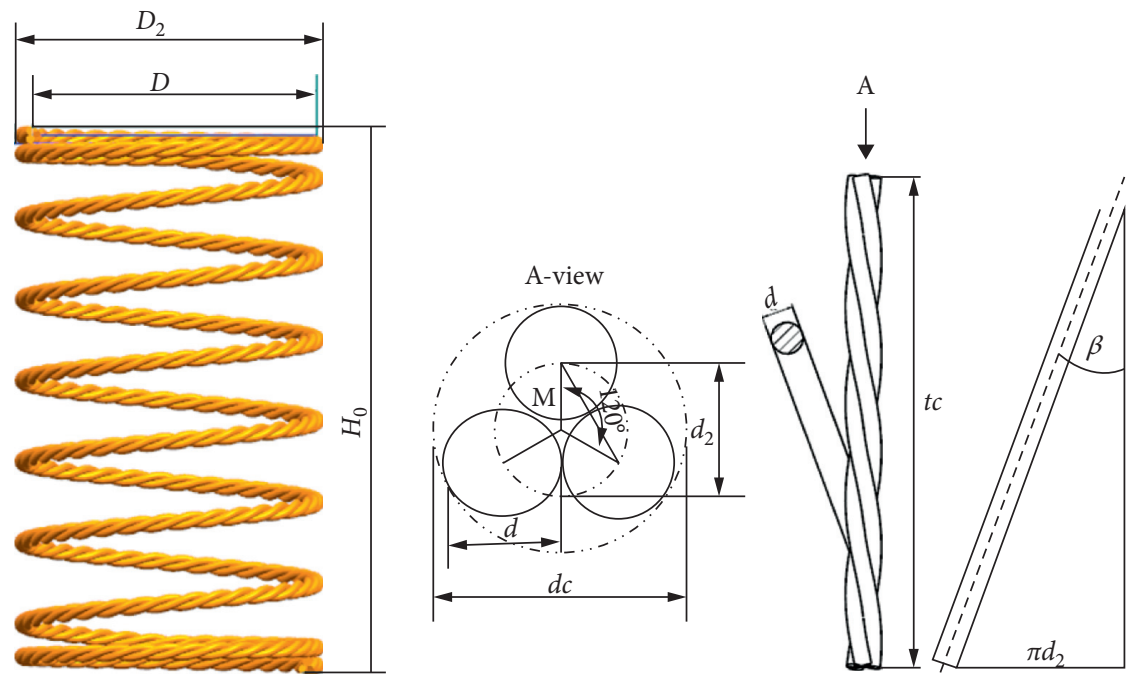

FIGURE 1: Geometric model of three-wire recoil spring.

And the relevant parameters of recoil spring are given in Table 1.

The center curve of each wire of the three-wire recoil spring is no longer an equal-pitch space helix like an ordinary cylindrically helical spring, but a secondary helix formed by rotating around the center curve of the strand, as shown in Figure 2. Therefore, the center curve of the strand and the center curves of the individual wires need to be drawn using a piecewise function.

Defining the helix angle of the center curve of strand as $\alpha$ and the polar angle as $\theta$, the equations of the center curve of strand can be expressed as

$$
\left\{\begin{array}{l}
x=\frac{D}{2} \cos \theta \\
y=\frac{D}{2} \sin \theta \\
z=\frac{D \theta}{2} \tan \alpha .
\end{array}\right.
$$

In Figure 2, the three components of vector $\overrightarrow{P Q}$ are written as

$$
\left\{\begin{array}{l}
\frac{d_{2}}{2} \cos \theta \cos \varphi+\frac{d_{2}}{2} \sin \alpha \sin \theta \sin \varphi \\
\frac{d_{2}}{2} \sin \theta \cos \varphi-\frac{d_{2}}{2} \sin \alpha \sin \theta \sin \varphi \\
\frac{d_{2}}{2} \cos \alpha \sin \varphi,
\end{array}\right.
$$

where $\varphi$ is the polar angle of the center curve of wire, and the center curve of wire equations are expressed as
TABle 1: Parameters of three-wire recoil spring.

\begin{tabular}{lc}
\hline Properties & Values \\
\hline$H_{0}(\mathrm{~mm})$ & 585 \\
$D(\mathrm{~mm})$ & 22 \\
$d(\mathrm{~mm})$ & 1.8 \\
Spring mass $(\mathrm{kg})$ & 0.235 \\
Number of wires & 3 \\
Pitch (mm) & 16.9 \\
$\beta\left(^{\circ}\right)$ & 30 \\
Active number of coils & 34 \\
Working length (mm) & 181 \\
Assembly length (mm) & 418 \\
Preload (N) & 180 \\
Working pressure $(\mathrm{N})$ & 340 \\
\hline
\end{tabular}

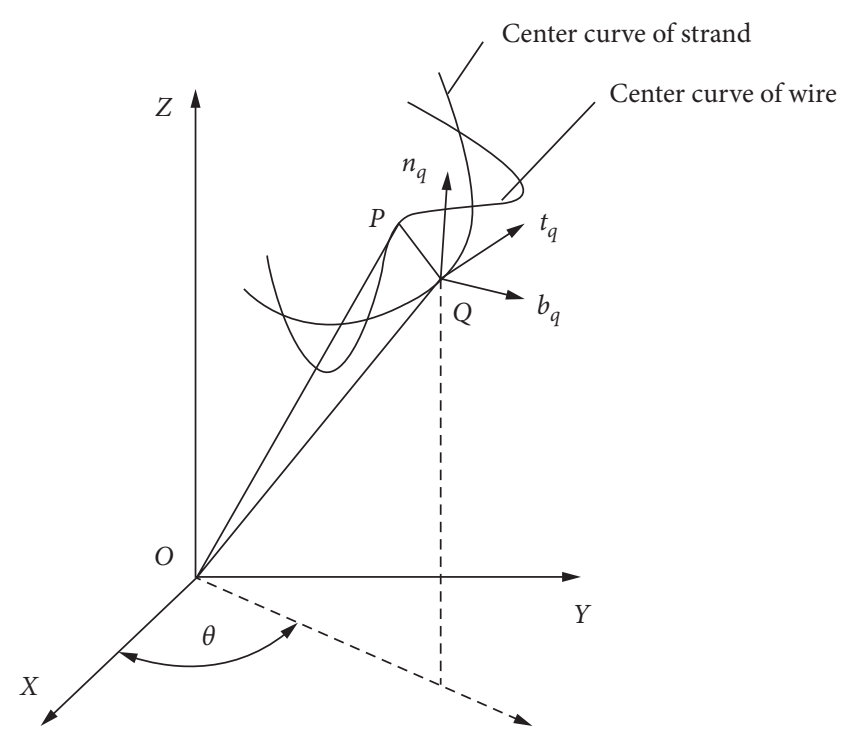

Figure 2: The center curves of strand and wire of recoil spring. 


$$
\left\{\begin{array}{l}
x=\left(r-\frac{d_{2}}{2} \cos \varphi\right) \cos \theta+\frac{d_{2}}{2} \sin \alpha \sin \theta \sin \varphi \\
y=\left(r-\frac{d_{2}}{2} \cos \varphi\right) \sin \theta-\frac{d_{2}}{2} \sin \alpha \cos \theta \sin \varphi \\
z=r \theta \tan \alpha+\frac{d_{2}}{2} \cos \alpha \sin \varphi
\end{array}\right.
$$

The number of helix turns of the center curve of wire around the center curve of strand is the wire helix multiple $c$, which can be determined by the following formula:

$$
c=\frac{\varphi}{\theta}=\frac{D}{d_{2} \cos \alpha \tan \beta} .
$$

Based on the geometric relationship between the parameters of the recoil spring model, we can get

$$
\begin{aligned}
d_{2} & =\frac{2 \sqrt{3}}{3} d, \\
\alpha & =\operatorname{arctg} \frac{t}{\pi d_{n n}},
\end{aligned}
$$

where $t$ is the pitch of the center curve of strand.

Then, the angle of rotation of the center curve of wire around the center curve of strand of the helix is

$$
\phi=c n=\frac{r}{d_{2} \cos \alpha \tan \beta} \cdot n=\frac{\sqrt{3} n r}{2 d \cos \alpha \tan \beta},
$$

where $n$ is the active number of coils of the center curve of strand.

The total number of coils of the multi-wire recoil spring can be written as

$$
n_{1}=n+n_{z}
$$

where $n_{z}$ is the number of coils of both ends of the recoil spring.

The free height of the recoil spring is expressed as

$$
H_{0}=n t+\left(n_{1}+1-n\right) d_{c}+2 \delta_{1} \text {, }
$$

where $\delta_{1}$ is the remaining gap in the state of maximum compression of the recoil spring.

2.2. Geometry Modeling of Three-Wire Recoil Spring. Based on the continuous multi-segment function in UG, the center curve of strand modeling of the three-wire recoil spring is performed, including 2 segments of space curves (free height of per segment is $298 \mathrm{~mm}$; active number of coils is 17), upper and lower closed space curves (effective height is $12 \mathrm{~mm}, 2$ closed ends), and the bridge line, as shown in Figure 3.

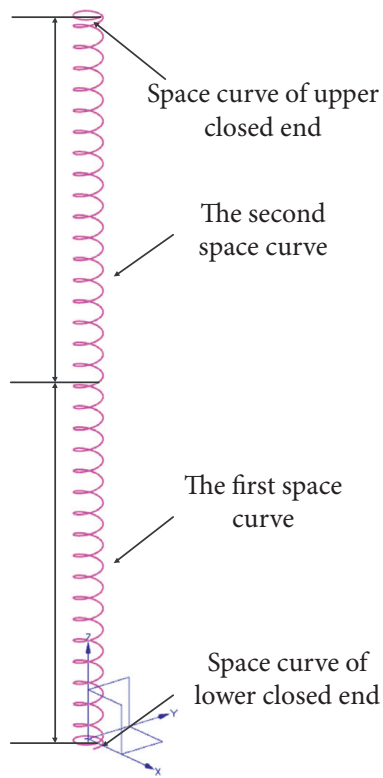

Figure 3: The center curve of strand of recoil spring.

The expressions for the sections of the center curve of strand are as follows:

The expressions of the lower closed curve are

$$
\left\{\begin{array}{l}
x t 3=\frac{D \cdot \cos (-(360-\mathrm{az}) \cdot \mathrm{nz} / 2 \cdot t-10)}{2} \\
y t 3=\frac{D \cdot \sin (-(360-\mathrm{az}) \cdot \mathrm{nz} / 2 \cdot t-10)}{2} \\
z t 3=-\mathrm{dc} \cdot \frac{\mathrm{nz}}{2} \cdot t .
\end{array}\right.
$$

The expressions of the first curve are

$$
\left\{\begin{array}{l}
x t 1=\frac{D \cdot \cos (\text { angle } \cdot n \cdot t)}{2}, \\
y t 1=\frac{D \cdot \sin (\text { angle } \cdot n \cdot t)}{2}, \\
z t 1=\text { height } \cdot t .
\end{array}\right.
$$

The expressions of the second curve are

$$
\left\{\begin{array}{l}
x t 2=\frac{D \cdot \cos (\text { angle } \cdot n \cdot t)}{2}, \\
y t 2=\frac{D \cdot \sin (\text { angle } \cdot n \cdot t)}{2}, \\
z t 2=2 \cdot \text { height } \cdot t .
\end{array}\right.
$$


The expressions of the upper closed curve are

$$
\left\{\begin{array}{l}
x t 4=\frac{D \cdot \cos ((360-\mathrm{az}) \cdot \mathrm{nz} / 2 \cdot t+360 \cdot n+10)}{2}, \\
y t 4=\frac{D \cdot \sin ((360-\mathrm{az}) \cdot \mathrm{nz} / 2 \cdot t+360 \cdot n+10)}{2}, \\
z t 4=\mathrm{dc} \cdot \frac{\mathrm{nz}}{2} \cdot t+\text { height, }
\end{array}\right.
$$

where $a z$ is the angle of the closed ends, $n z$ is the number of closed ends, $\mathrm{dc}=d / \cos 30^{\circ}+d$, height $=H_{0}-(n z+1) \cdot d c$ is the height of the middle active coils, $n$ is the active number of middle coils of the spring, and angle $=360^{\circ}$ is the corresponding angle for each coil.

To obtain a three-dimensional model of the multi-wire recoil spring, first, in a plane perpendicular to the center curve of the strand, establish three straight lines with an included angle of $120^{\circ}$. Then, based on the angle rotation rule of the center curve of wire around the center curve of strand established in Section 2.1, the 3 straight lines are swept along the center curve of strand to generate three helix surfaces, and their outer contours are the center curves of three wires. Further at the end of the center curves of the wires, create three swept circles. Finally, the sweeping circles are swept along the center curves of wires to obtain a three-dimensional model of the multi-wire recoil spring, as shown in Figure 4.

\subsection{Parametric Modeling of Multi-Wire Recoil Spring.} Based on the above-mentioned multi-wire recoil spring center curves of strand and wires equations and spring geometric parameters calculation method, first, drive parameters of recoil spring are defined in the UG by expressions, as shown in Table 2. Then, the calculation expressions of other geometric parameters of recoil spring are established, such as the space curve law of the center curve of wire and the twist angle of strand and the pitch. For the corresponding expression calculation formulas, please refer to Sections 2.1 and 2.2, and we will not repeat them here, so as to establish a parametric template for recoil spring.

Further based on the multi-wire recoil spring parameterization template, use the secondary development technology of Menu Script and UIstyler to design the UG menu bar and UI dialog box, and create a $\mathrm{C}++$ program through the UG/Open API to implement the modification of the size expressions of the multi-wire recoil spring parameterized template through the UI dialog box, and change the spring parameterized template to achieve the multi-wire recoil spring parameterized modeling.

\section{Analysis of Impact Characteristics of Recoil Spring}

3.1. Working Condition Analysis of Recoil Spring. The recoil spring is assembled in the piston cylinder and is sleeved on the outside of the piston rod. Its rear end is pressed against the annular base of the piston cylinder, and the front end is pressed on the guide stem of the piston rod with preload $F_{1}$. After the bullet is fired, the gunpowder gas pushes the warhead forward. When the bullet passes through the air hole, part of the gunpowder gas flows into the air chamber through the air hole. Due to the impact and expansion of the high-temperature and highpressure gunpowder gas, the piston is pushed, driving the coupling sleeve and the bolt carrier, so that the entire movable mechanism enters the recoil process. The recoil spring is compressed during the recoil process of the moving parts to reserve energy for its re-entry. The recoil process is completed when the moving parts hit the buffer device, and the moving parts rebound to get the initial receding speed. At the same time, the recoil spring began to stretch, pushing the piston forward under the action of the spring force, and the re-entry action of the moving frame of bolt carrier is performed through the coupling sleeve.

The recoil spring mainly bears the high-speed impact of the automaton on its end during the recoil and re-entry process. It has the characteristics of short duration and fast spring deformation. In the recoil process, the recoil spring stress changes drastically, and it is possible to exceed the elastic limit of the material. When re-entry is started, the stress of the recoil spring changes from compressive stress to tensile stress instantly, and the continuous repetition of dynamic stress caused by high-speed alternating load easily causes fatigue failure of recoil spring. Based on the operating conditions of the recoil spring, an internal trajectory calculation model and an air chamber pressure calculation model are established to obtain the law of the change in the bore pressure and the air chamber pressure when the gun fires, and the dynamics simulation of the automata mechanism is applied to accurately obtain the speed change law of the recoil process of the moving parts, as shown in Figure 5.

The characteristic curve of recoil spring is shown in Figure 6. In the figure, $H_{0}$ is the free length of the recoil spring, $H_{1}$ is the assembled length of the recoil spring, $F_{1}$ is the preload, $\mathrm{H}_{2}$ and $F_{2}$ are the length and spring force when recoil spring recoil moves to the right position, respectively, and $H_{3}$ and $F_{3}$ are the length and force when the spring coils are bonded to each other, respectively.

\subsection{Finite Element Model of Impact Characteristics of Recoil} Spring. Finite element method is widely used in stress analysis of springs $([1,9,13,14])$. This paper uses ABAQUS to construct a finite element simulation model of recoil spring impact characteristics in accordance with the multi-wire recoil spring characteristic curve and actual operating conditions, to analyze the dynamic characteristics of displacement, velocity, acceleration, stress, and strain of recoil spring under impact load.

To simplify the simulation model, the bolt carrier is replaced with a cylinder of equal mass, and a three-dimensional assembly model of recoil spring, guide rod, and bolt carrier is established using UG, as shown in Figure 7, where the mass of the cylinder is $2.3 \mathrm{~kg}$. Then, import the assembly model into Abaqus.

The material of recoil spring is $55 \mathrm{Si} 2 \mathrm{Mn}$, which is suitable for working under high stress. The material property curve (Figure 8) and parameters (Table 3) are obtained through the material mechanical property test. 

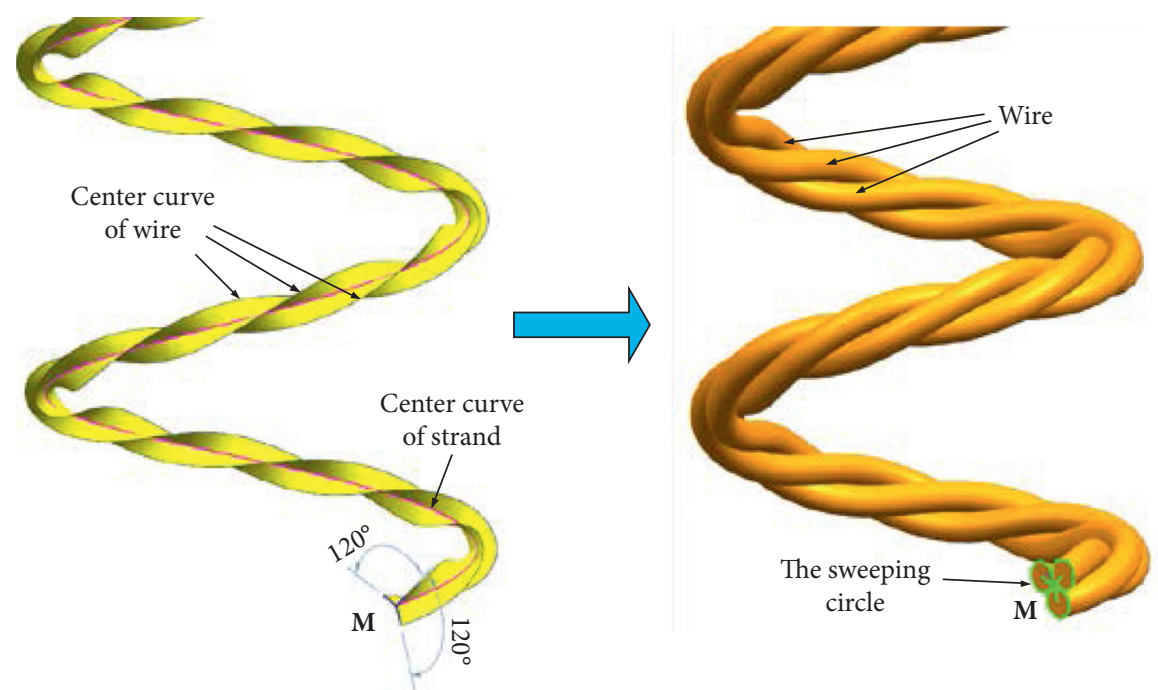

FIgURE 4: Schematic diagram of the 3D modeling process of three-wire recoil spring.

TABLE 2: Driving parameters of three-wire recoil spring.

\begin{tabular}{lc}
\hline Parameters & Expressions \\
\hline$D(\mathrm{~mm})$ & spring_dia $=22$ \\
$d(\mathrm{~mm})$ & spring_d $=1.8$ \\
Active number of coils & spring_ $n 1=34$ \\
Number of closed ends & Spring_ $n z=2$ \\
$H_{0}(\mathrm{~mm})$ & spring_H$H_{0}=585$ \\
Remaining gap $(\mathrm{mm})$ & spring_delta $=0.5$ \\
\hline
\end{tabular}

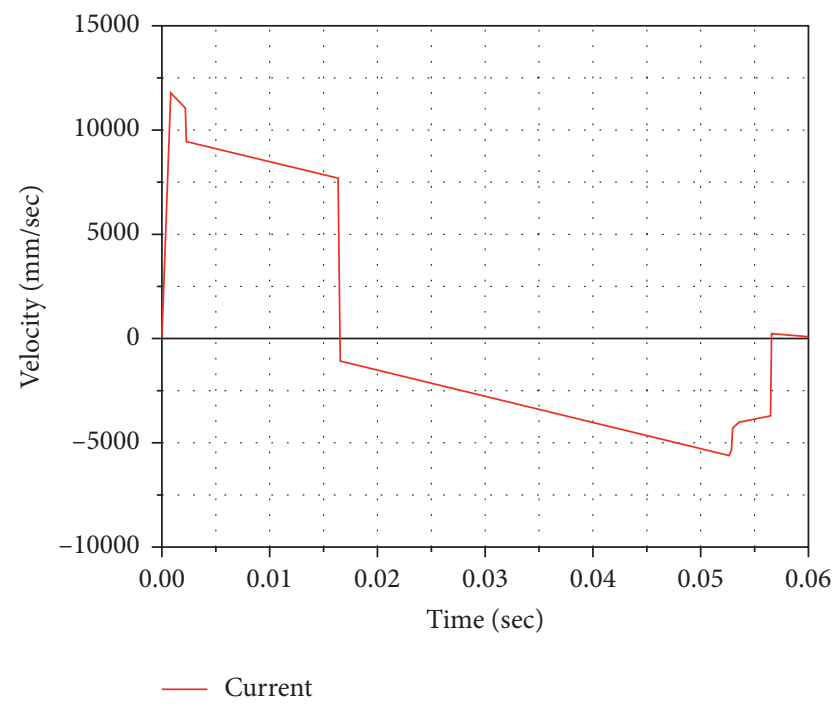

Figure 5: Bolt carrier speed curve.

Recoil spring is defined as an elastoplastic body. To avoid hourglass mode and volumetric locking, a linear non-coordinated solid unit C3D8R is used to mesh it. The unit size is set to $0.2 \mathrm{~mm}$, the number of units is 161760 , and the number of nodes is 364230 . And define the guide rod and simplified bolt carrier as discrete rigid bodies. The meshing of the assembly model of the recoil spring, guide rod, and simplified bolt carrier is shown in Figure 9.

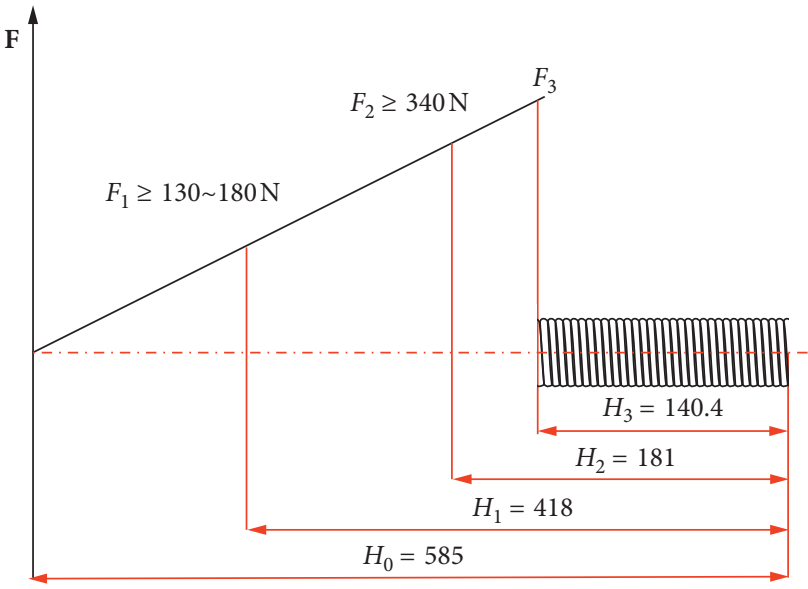

Figure 6: The characteristic curve of recoil spring.

Apply load in three load steps according to recoil spring characteristic curve and actual working conditions. The first load step is the preloading stage. Considering the recoil spring assembly preloading process, the relationship between displacement and time is defined in Table 4. The second load step is the recoil stage. Based on the recoil spring recoil process, the relationship between the speed and time of the bolt carrier is defined according to Figure 5, as shown in Table 4. The third load step is the re-entry stage, and the relationship between the speed and time of the bolt carrier is also defined according to Figure 5, as shown in Table 4.

Apply full constraints to the guide rod, define the bolt carrier to slide freely in the direction of the guide rod, constrain the other 5 degrees of freedom, and set by binding nodes. The friction coefficient between the recoil spring wires is set to 0.1 [7], and the friction coefficient between the recoil spring and bolt carrier and guide rod is set to 0.08 .

3.3. Finite Element Analysis Results of the Impact Characteristics of the Recoil Spring. Figure 10 shows the stress distribution when the stress wave is transmitted to the 1st coil (the 


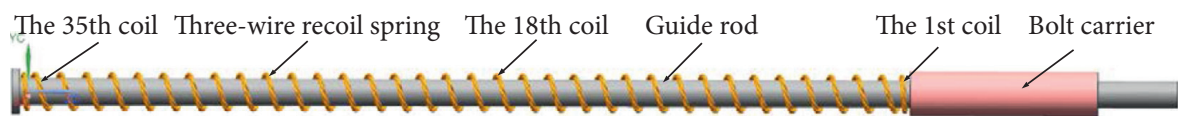

FIGURE 7: 3D assembly model of recoil spring, guide rod, and bolt carrier.

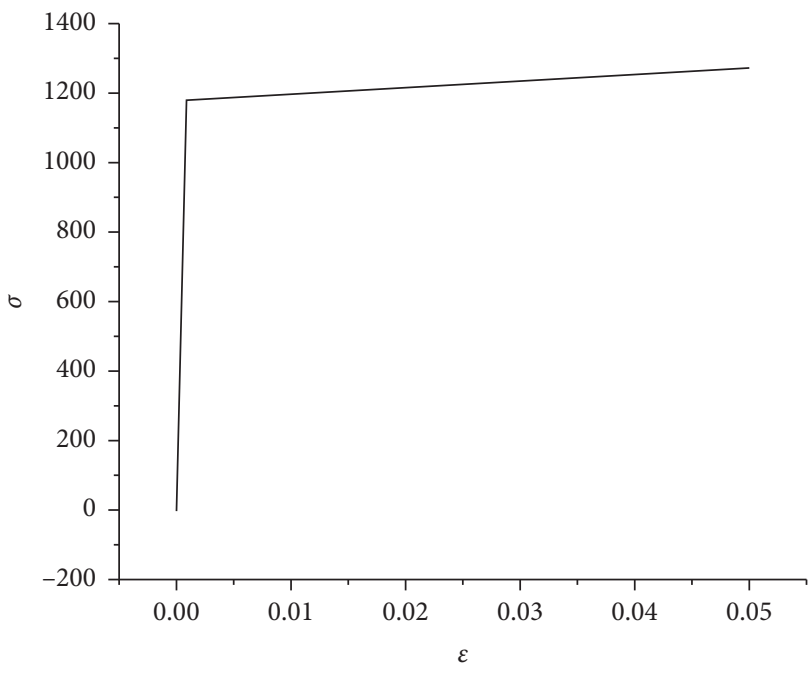

FIGURE 8: 55Si2Mn material performance curve.

TABle 3: Material parameters of 55Si2Mn.

\begin{tabular}{lccccccc}
\hline Properties & $\begin{array}{c}E \\
(\mathrm{Gpa})\end{array}$ & $M$ & $\begin{array}{c}\rho(\mathrm{kg} / \\
\left.\mathrm{m}^{3}\right)\end{array}$ & $\begin{array}{c}\sigma_{s} \\
(\mathrm{Gpa})\end{array}$ & $\begin{array}{c}\sigma_{b} \\
(\mathrm{GPa})\end{array}$ & $\begin{array}{c}\delta \\
(\%)\end{array}$ & $\psi$ \\
\hline Values & 207 & 0.27 & $7.73 e 3$ & 1.177 & 1.275 & 5 & 30 \\
\hline
\end{tabular}

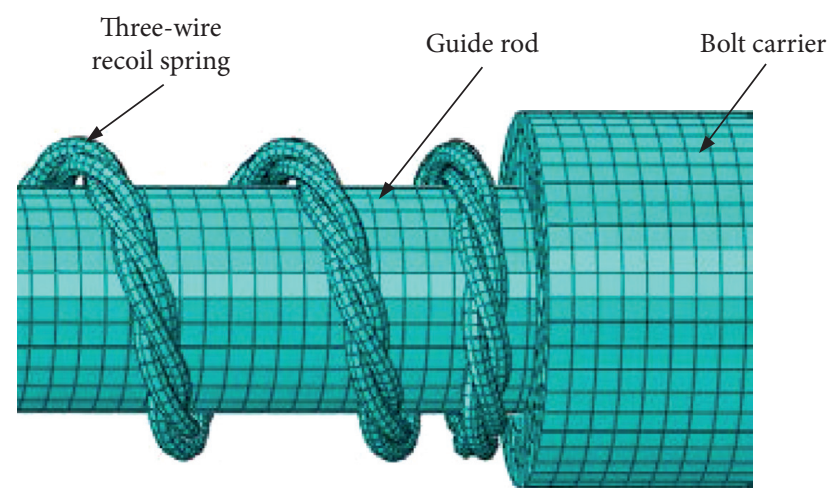

FIgURE 9: Meshing of recoil spring, guide rod, and bolt carrier.

free end or impact end), 18th coil (the middle coil), and 35th coil (the fixed end) of recoil spring during the multi-wire recoil spring recoil process obtained by simulation. It can be seen from Figure 10 that, during the recoil, the stress reaches a maximum value of $1518 \mathrm{MPa}$ at the point $\mathrm{P} 1$ of the 1st coil. After the bolt carrier hits the recoil spring, the stress is transmitted in the coils in the form of waves. Each coil is no longer deformed uniformly and trembles. The stress waves cause the coils to squeeze each other during the transmission process, which increases the contact stress. The reason is that the direction in which the wires of the spring are twisted is opposite to the direction in which the spring is wound. The
TABle 4: Load steps of different stages of recoil spring.

\begin{tabular}{lccc}
\hline Load steps & Properties & Values & Values \\
\hline \multirow{2}{*}{ Preloading stage } & Time $(\mathrm{s})$ & 0 & 0.03 \\
& Displacement $(\mathrm{mm})$ & 0 & 177 \\
Recoil stage & Time $(\mathrm{s})$ & 0 & 0.026 \\
& Speed $(\mathrm{m} / \mathrm{s})$ & 9 & 7 \\
Re-entry stage & Time $(\mathrm{s})$ & 0 & 0.066 \\
& Speed $(\mathrm{m} / \mathrm{s})$ & 1.1 & 6.1 \\
\hline
\end{tabular}

greater the amount of compression of the spring, the tighter the wires are twisted, and the greater the frictional resistance caused by the contact stress. When recoil spring is impacted by the bolt carrier, the stress in the coil is transmitted to the fixed end in the form of a compression wave, and reflection occurs at the fixed end. The displacement of each particle of the coil is the sum of the compression wave displacement and the reflected wave displacement, and when the impact speed is high, the coils will squeeze each other.

Figure 11 shows the stress contours during recoil spring reentry. Due to the residual stress wave during the recoil process, the compression of the coil at the fixed end of the spring at the initial stage of the re-entry is increased, and the stress continues to increase. After 0.006 seconds, the maximum stress at the point $\mathrm{P} 3$ of fixed end reaches $1531 \mathrm{MPa}$. Then, due to the spring force and the reflection of the stress wave at the fixed end, the compression phenomenon of the coil is relieved and the stress is reduced. After $0.02 \mathrm{~s}$, the stress value at the middle position of the recoil spring is reduced to $1372 \mathrm{MPa}$. By analyzing the entire recoil and re-entry process, it can be known that the overall stress due to the superposition of stress waves during the re-entry process is significantly greater than the stress generated during the recoil process, and the maximum stress is located at the fixed end, which indicates that recoil spring is most likely to have fatigue failure at the fixed end.

Figure 12 shows the time displacement response curves of the 1st coil, 18th coil, and 35th coil of recoil spring after being impacted. It can be seen from the figure that the displacement is no longer linearly distributed in the axial direction, and the displacement of the fixed end is smaller than the free end.

Figure 13 is the velocity response curve of the 1st coil, 18 th coil, and 35 th coil after recoil spring is impacted. It can be seen from the figure that, after the recoil spring is hit by the bolt carrier, the speed of the free end increases rapidly. As the recoil spring is continuously compressed, the speed propagates to the fixed end and gradually decreases. In this high-speed impact, the impact energy received by the free end of recoil spring is transmitted to the fixed end in the form of longitudinal waves and is reflected at the fixed end and the free end. In addition to the resonance of the spring itself, the frictional resistance generated by the mutual extrusion and sliding between the wires of the recoil spring, 


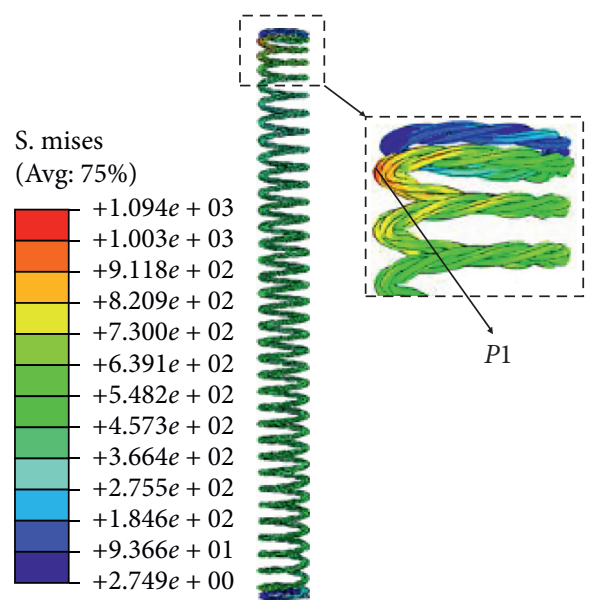

Step: step-3

Increment 16923: step time $=1.4813 E-03$

Primary var: S. mises

Deformed var: $\mathrm{U}$ deformation scale factor: $+1.000 e+00$

S. mises

(Avg: $75 \%)$

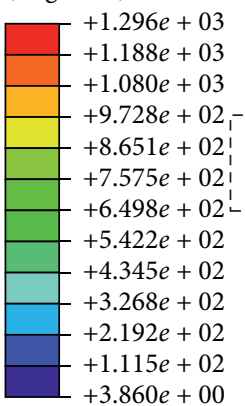

Step: step-3

Increment 169189: step time $=1.4813 E-02$

Primary var: S. mises

Deformed var: $\mathrm{U}$ deformation scale factor: $+1.000 e+00$

(a)

(b)

S. mises

(Avg: $75 \%)$
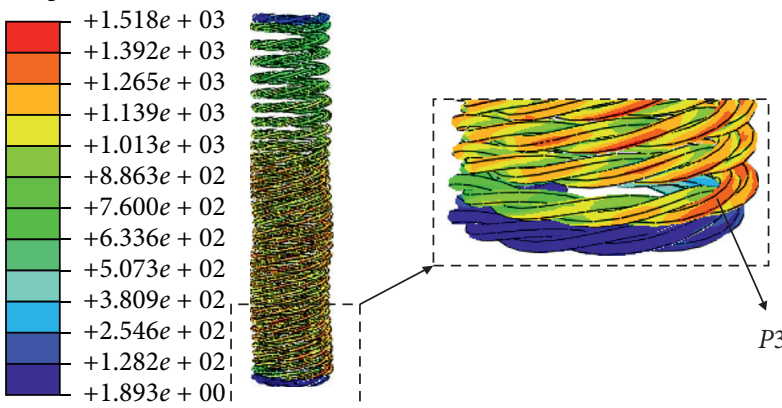

Step: step-3

Increment 338350: step time $=2.9625 E-02$

Primary var: S. mises

Deformed var: $\mathrm{U}$ deformation scale factor: $+1.000 e+00$

(c)

FIgURE 10: Stress contours of recoil spring at different moments during recoil. (a) $t=0.0015 \mathrm{~s}$. (b) $t=0.015 \mathrm{~s}$. (c) $t=0.029 \mathrm{~s}$.

and many other factors all have an impact on the internal motion of recoil spring.

\section{Impact Characteristics Experiment of Recoil Spring}

To verify the finite element simulation results of recoil spring impact characteristics, a recoil spring impact characteristic experiment was performed. The time-varying changes of dynamic parameters such as speed and displacement during recoil spring recoil and re-entry are compared with the finite simulation results.

4.1. Experiment Device. Based on the recoil spring characteristic curve and actual working conditions, a set of recoil spring impact characteristic experiment equipment was designed, as shown in Figures 14 and 15.
The recoil spring is sleeved on the guide rod, the fixed end is topped on the support base, and the free end is connected with the baffle. The support base realizes the assembly limit, the baffle, the support base, and the rubber gasket realize the recoil movement of the firearm automaton to the correct position, and then the impact buffer device rebounds and starts to re-enter. The steel cylinder simulates a bolt carrier with a mass of $2.3 \mathrm{~kg}$. The impact load of the steel cylinder on the recoil spring is provided by the power spring. The steel cylinder compresses the power spring to store energy. After release, the potential energy of the spring is converted to the kinetic energy of the steel cylinder. The amount of compression of the power spring can control the impact speed of the steel cylinder on the recoil spring. The laser rangefinder is used to obtain the change of the free end displacement of recoil spring with time, and the high-speed camera is used to obtain the dynamic changes of recoil spring displacement and speed during recoil and re-entry. 


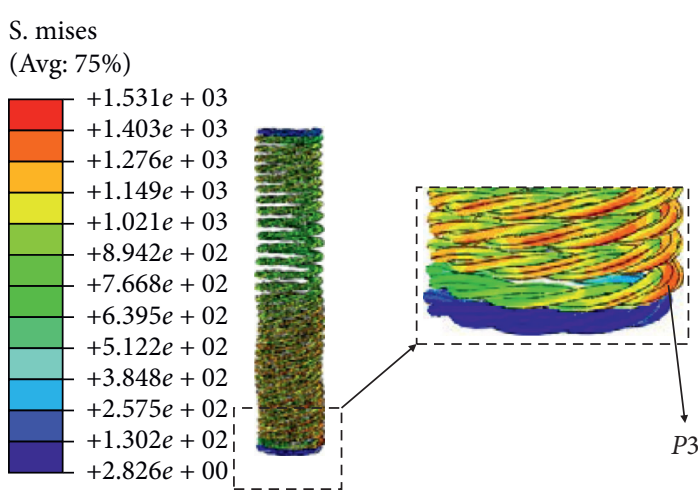

Step: step- 4

Increment 75399: step time $=6.6001 E-03$

Primary var: S. mises

Deformed var: $\mathrm{U}$ deformation scale factor: $+1.000 e+00$
S. mises

(Avg: 75\%)

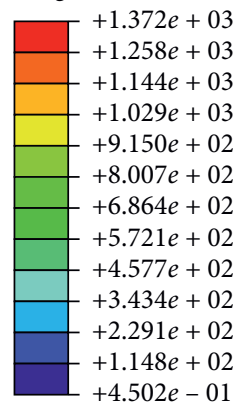

Step: step-4

Increment 226180: step time $=1.9800 E-02$

Primary var: S. mises

Deformed var: $\mathrm{U}$ deformation scale factor: $+1.000 e+00$

(a)

(b)

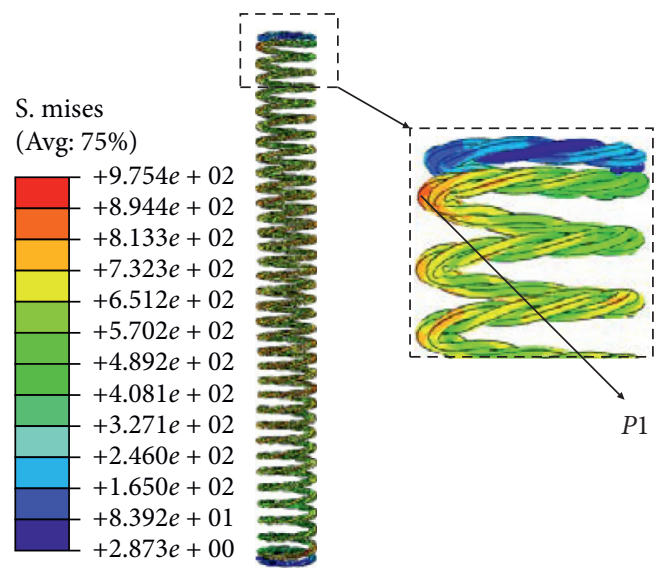

Step: step- 4

Increment 567677: step time $=4.9500 E-02$

Primary var: $S$. mises

Deformed var: $\mathrm{U}$ deformation scale factor: $+1.000 e+00$

(c)

Figure 11: Stress contours of recoil spring at different moments during re-entry. (a) $t=0.006 \mathrm{~s}$. (b) $t=0.02 \mathrm{~s}$. (c) $t=0.05 \mathrm{~s}$.

4.2. Impact Characteristics Experiment Measurement Principle. The inner wall of the power spring and the steel cylinder is relatively smooth, so the influence of the friction between the power spring and the guide rod and between the steel cylinder and the guide rod on the impact speed can be ignored. The relationship between the impact speed of the steel cylinder on the recoil spring and the compression of the power spring satisfies the conservation of kinetic energy:

$$
\frac{1}{2} m v^{2}=\frac{1}{2} k x^{2}
$$

where $m$ is the mass of the steel cylinder, $v$ is the speed of the steel cylinder, $k$ is the stiffness of the power spring, and $x$ is the amount of compression of the power spring.

By using the laser rangefinder, the displacement-time curve of the 1st coil, 18 th coil, and 35 th coil of recoil spring can be measured during recoil and re-entry. Then, derivate it with respect to time to obtain the speed-time curve.

The high-speed camera can realize non-contact measurement of parameters such as displacement, speed, acceleration, etc. It has the advantages of convenient test arrangement, high measurement accuracy, strong reliability, and reusability. In the pictures taken by the high-speed camera, the time interval between each two adjacent frames is determined, and the position of the subject in each picture can be determined according to the scale in the picture, so as to obtain the dynamic displacement relationship of the subject. Because the time interval between two adjacent frames is very small, and the distance the object moves is also very short, the average speed of the object within this distance can be approximated as the instantaneous speed of the object at a certain moment. The average speed solution formula is expressed as follows: 


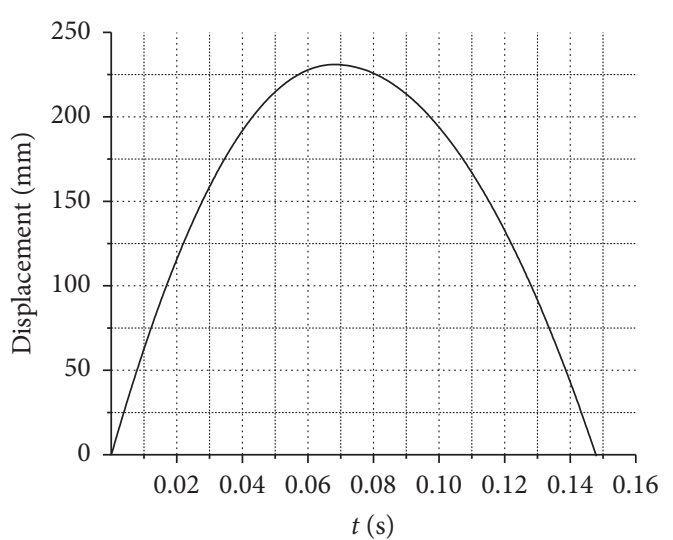

(a)

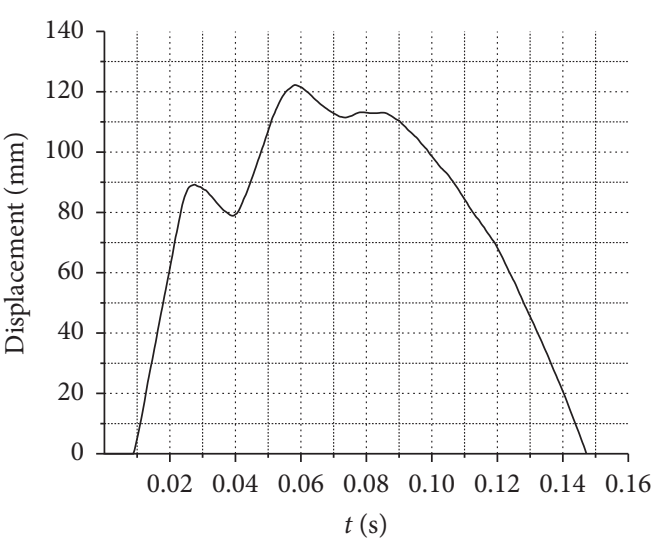

(b)

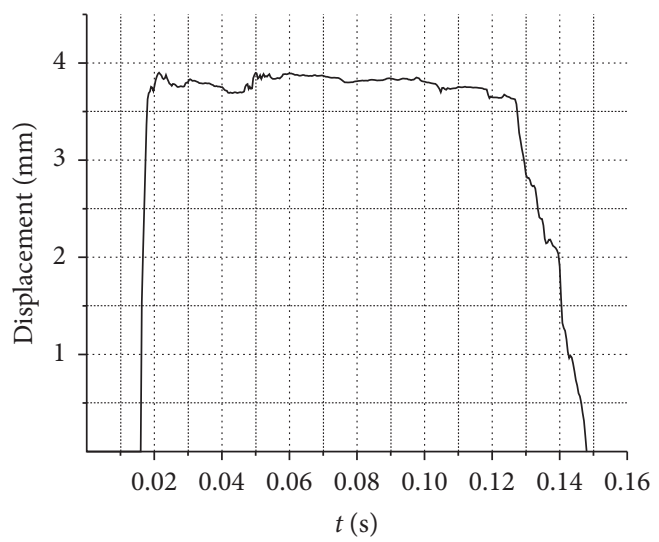

(c)

Figure 12: Displacement-time curves of different coils. (a) The 1st coil. (b) The 18th coil. (c) The 35th coil.

$$
v=\frac{l_{n}-l_{0}}{t_{n}-t_{0}}=\frac{N\left(l_{n}-l_{0}\right)}{n},
$$

where $l_{0}$ is the position of the object to be measured at time $t_{0}, l_{n}$ is the position of the object to be measured at time $t_{n}, N$ is the number of high-speed photographing frames, and $n$ is the number of frames from $t_{0}$ to $t_{n}$.

Therefore, the high-speed camera can be used to measure the relationship between the displacement and time of different coils under high-speed impact loads. Then, differentiate it with time, so that the relationship between the speed and time of different coils can be obtained.

\subsection{Comparison of Simulation and Experimental Results.} Figures 16-21 show the comparison of the simulation results with the experimental results of impact characteristics of different coils of three-wire recoil spring. It can be seen from Figures 16 and 17 that the simulation results of the displacement and velocity-time history curves of the 1st coil (free end) differ little from the experimental values. In addition, as can be seen from Figures 18 and 19, the simulation results of the displacement and velocity-time history curves of the 18th coil are basically consistent with the experimental values trends. From Figures 20 and 21, it can be seen that the simulation results of the displacement and velocity-time history curves of the 35th coil (fix end) are consistent with the overall trend of the test data, but the error between the simulation results of the displacement-time history curve and the experiment is slightly larger, mainly because the 35th coil has a small displacement during the recoil spring recoil and re-entry process, which causes measurement difficulties and increases the measurement result error.

From the comparison of the simulation results and experimental results of the displacement and speed-time history curves of different coils, it can be seen that the trends of the different coil displacement and speed-time history curves are in good agreement with the experimental results. The maximum error between the simulation value and the experimental value of the maximum displacement of the coils is $4 \%$, and the minimum is only $0.6 \%$. The maximum error between the simulation value and the experimental value of the maximum speed of the coils is $8 \%$, and the minimum is $5 \%$. In general, the impact characteristics of different spring coils of multi-wire recoil spring are in good agreement with the experimental results, indicating that the adopted finite element simulation 


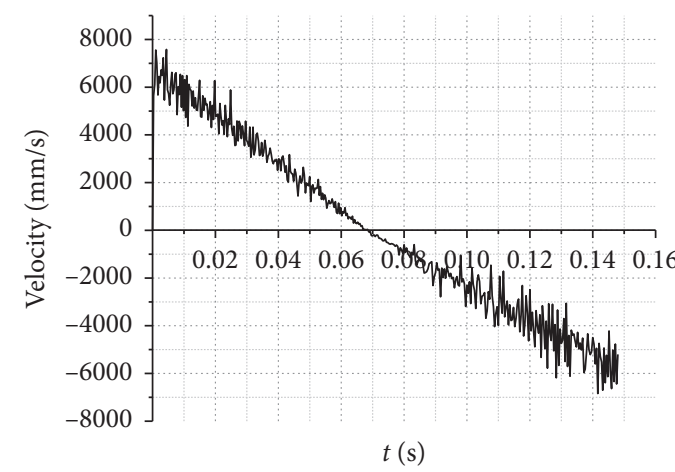

(a)

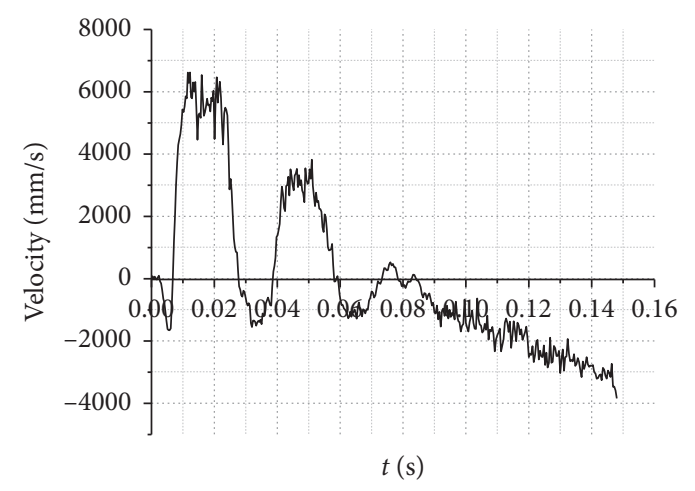

(b)

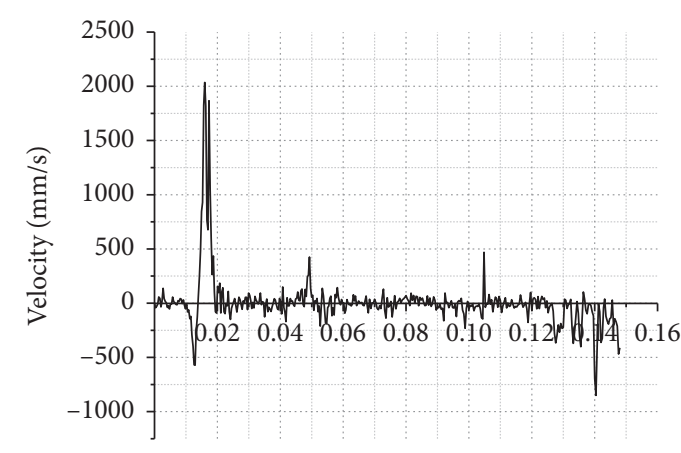

$t(\mathrm{~s})$

(c)

Figure 13: Speed-time curves of different coils. (a) The 1st coil. (b) The 18th coil. (c) The 35th coil.

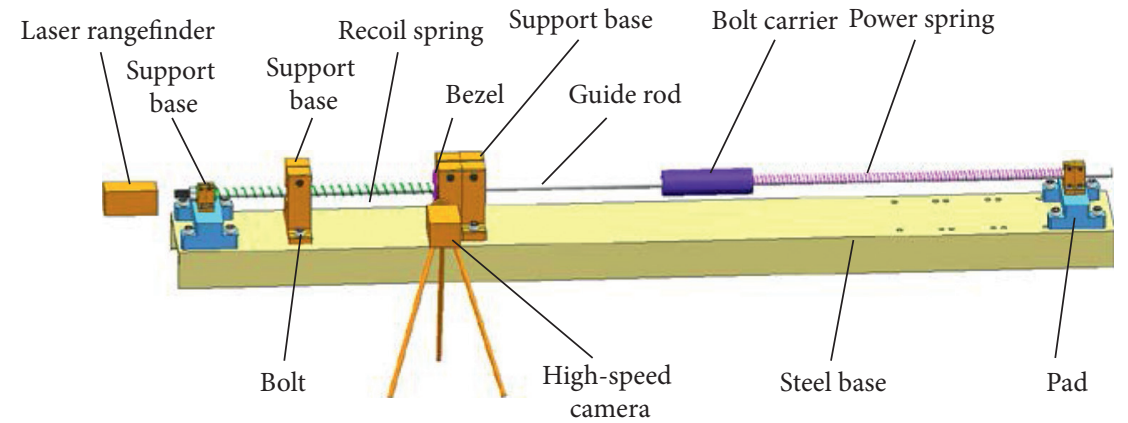

FIGURE 14: Schematic diagram of recoil spring impact characteristic experiment device.

method of recoil spring impact characteristics can better reflect the dynamic response characteristics of recoil spring under the impact of bolt carrier.

\section{Fatigue Life Prediction}

5.1. Time Domain Analysis of Impact Stress of Recoil Spring. Select three maximum stress points $\mathrm{P} 1, \mathrm{P} 2$, and $\mathrm{P} 3$ at the free end (impact end), middle coil, and fixed end of the spring, respectively, that is, the dangerous points in the three stress propagation stages. The stress-time history curve of the three dangerous points is shown in Figure 22. The stress-time history curve at the P3 of the fixed end shows that the stress value of the recoil spring reaches the maximum after the stress wave reaches the fixed end, and the curve does not decrease, indicating that the stress wave is not reflected at the fixed end. From the stress-time history curve of P1 of the impact end, it can be seen that the stress change at this location is more dramatic. Therefore, it can be obtained that the position of maximum stress $\mathrm{P} 3$ and the position of the most severe stress change P1 are most prone to fatigue.

5.2. Fatigue Stress Spectrum of Recoil Spring. The stress-time history curve of the dangerous points $\mathrm{P} 1$ and P3 in the impact process of the recoil spring mentioned above is a continuous random process, which cannot be directly used to determine its final stress spectrum. The invalid stress in the response process must be eliminated, that is, the stress value with a small amplitude is removed by the compression process, and the effective amplitude value, the mean value, and the number of cycles are determined by statistical 


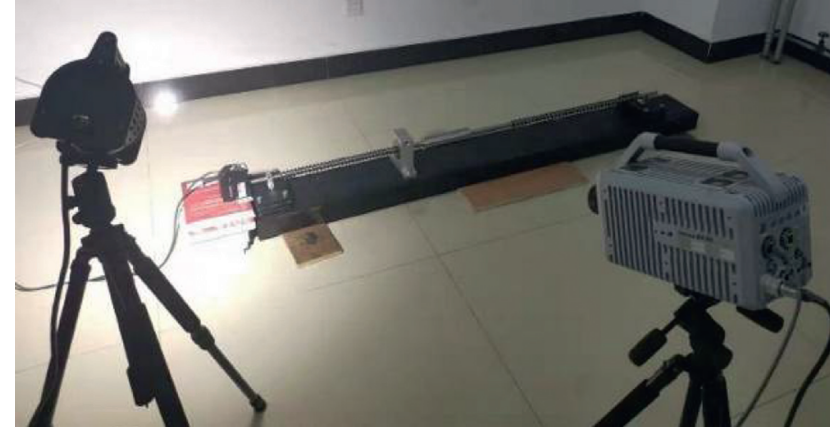

FIgURE 15: Impact characteristics experiment device for recoil spring.

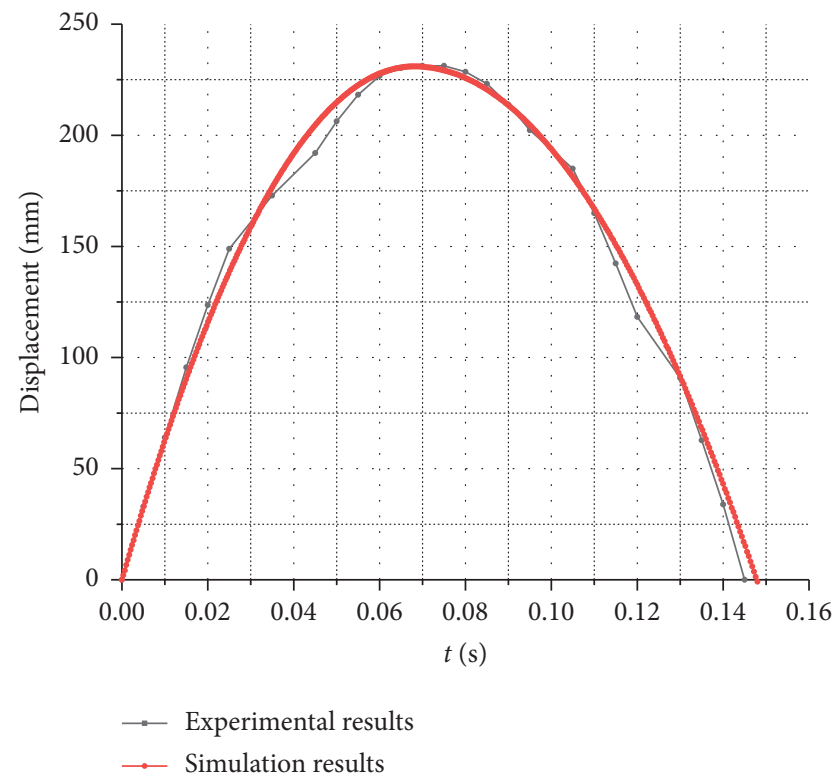

Figure 16: Comparison of the displacement-time history curves of the 1st coil.

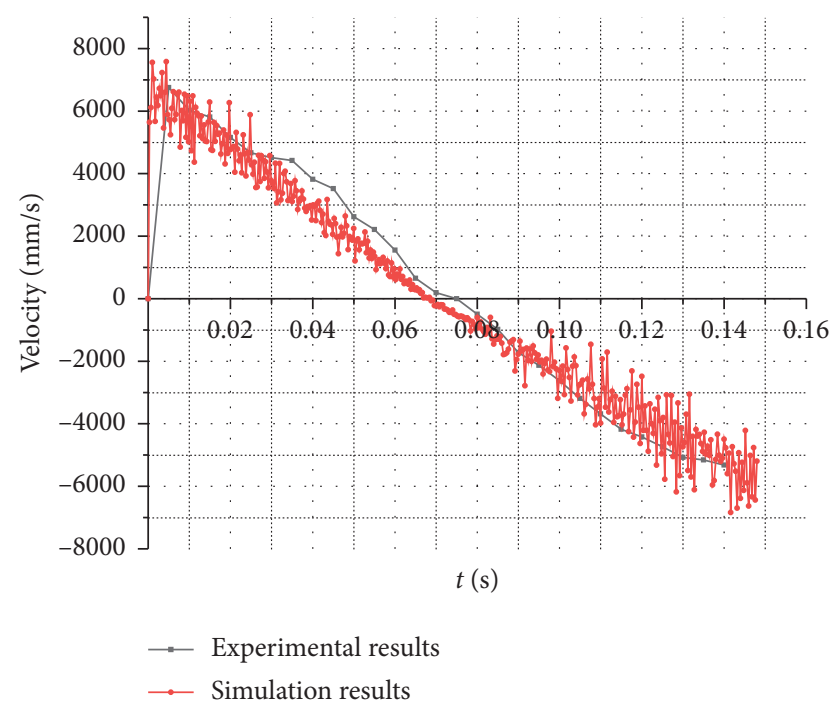

FIGURE 17: Comparison of the velocity-time history curves of the 1st coil.

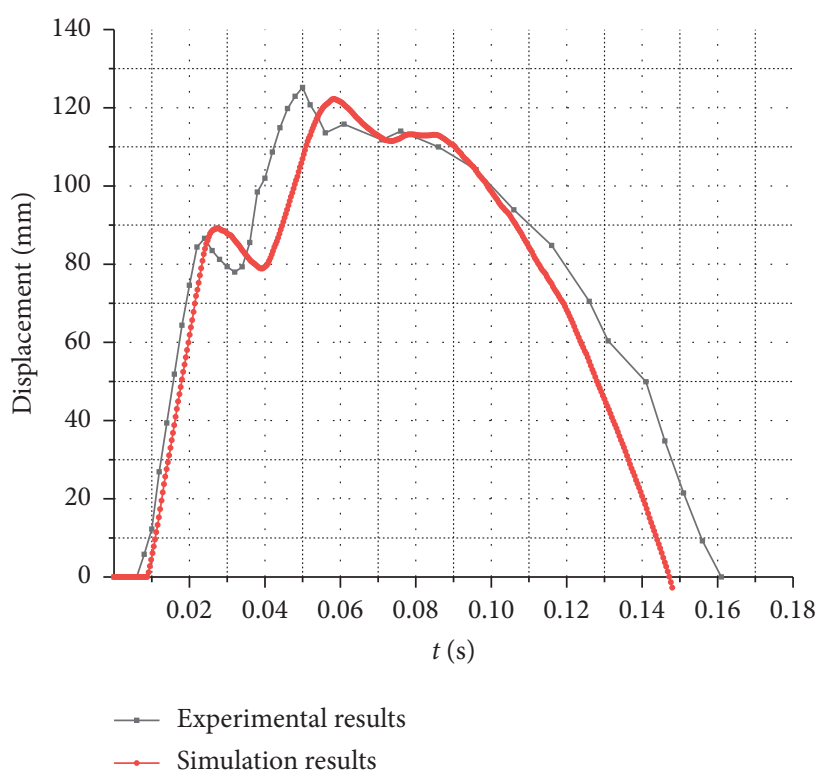

FIGURE 18: Comparison of the displacement-time history curves of the 18th coil.

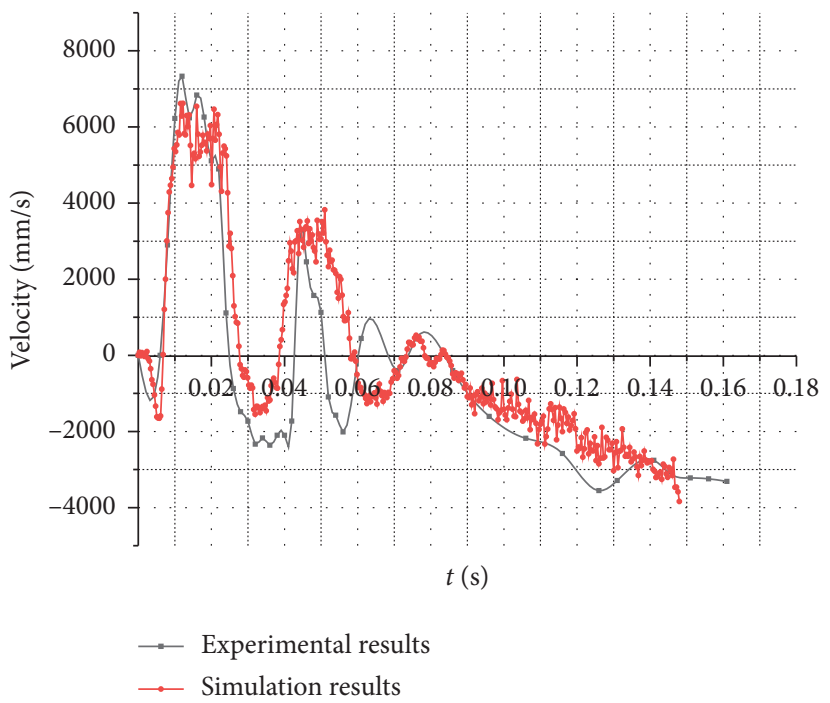

FIgURE 19: Comparison of the velocity-time history curves of the 18th coil.

calculation method to obtain the final effective stress spectrum. In this paper, the rain flow counting method is used to perform statistical calculation on the time history curve of recoil spring, and the stress spectrum is represented by discrete stress cycles. The final result is expressed by the stress amplitude and the mean value of stress [15].

5.2.1. Compression of Curves. Compression processing includes steps such as sampling, peak-valley detection, and omission of invalid amplitude values.

In this paper, the sampling frequency of recoil spring is determined to be $500 \mathrm{~Hz}$ according to the time history and the excitation frequency. The invalid amplitude limit of 


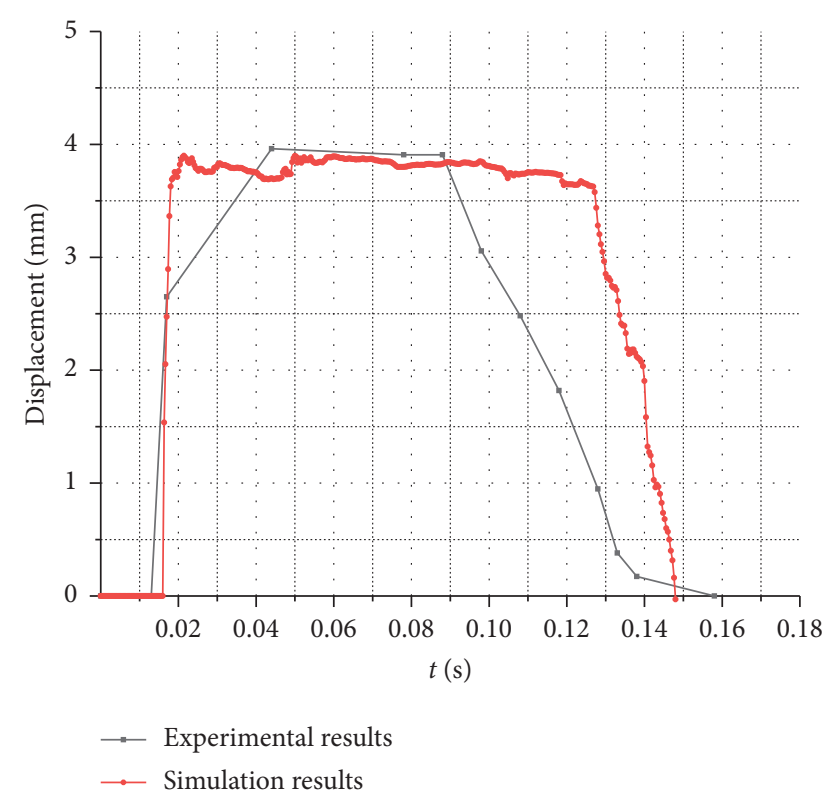

Figure 20: Comparison of the displacement-time history curves of the 35 th coil.

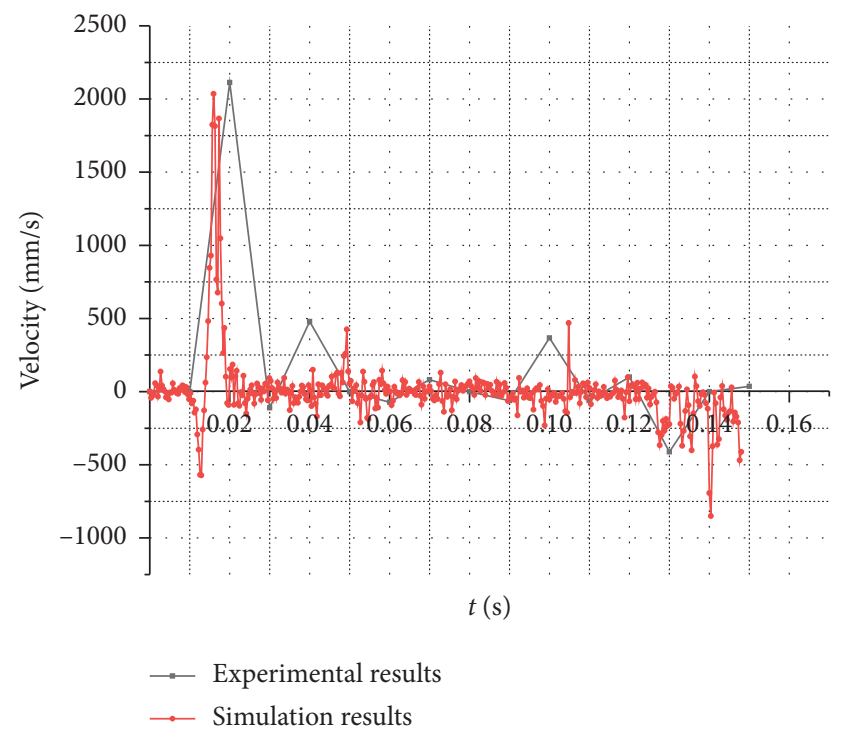

FIGURE 21: Comparison of the velocity-time history curves of the 35th coil.

recoil spring is determined by the range threshold formula, which is expressed as

range thershold $=($ maximum value - minimum value $) \times \Delta$,

where $\Delta$ is the threshold accuracy, and its value is $5 \%$.

According to equation (15), the range threshold of the point $\mathrm{P} 1$ at the free end is $33 \mathrm{MPa}$. The effective stress-time history curve after removing the invalid stress point is shown in Figure 23. It can also be obtained that the range threshold of the point $\mathrm{P} 3$ at the fixed end is $42.8 \mathrm{MPa}$, and the effective

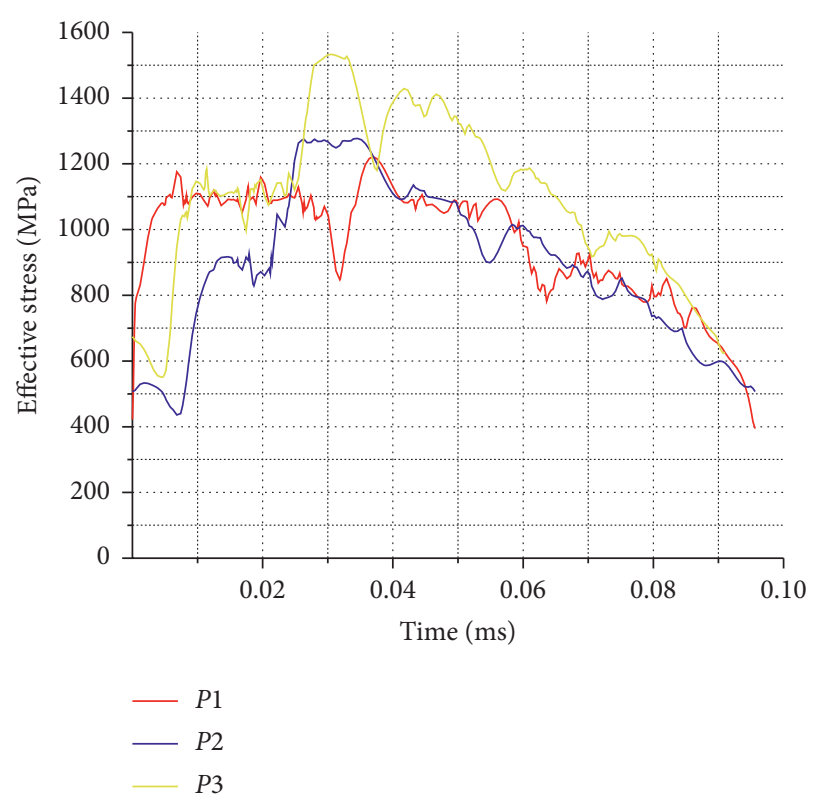

FIGURE 22: The stress-time history curve of the three dangerous points.

stress-time history curve after removing the invalid stress point is shown in Figure 24.

5.2.2. Rain Flow Counting Method. The main steps of rain flow counting method are as follows [16]:

(1) Rearrange fatigue stress-time history starting from the highest peak or lowest valley.

(2) The rain current flows down from the inside of each peak (valley) in turn, falls at the next valley (peak), and stops at a peak value higher than the initial value.

(3) Stop when encountering the rain flowing down.

(4) Record the number of cycles, mean stress, and amplitude.

The effective stress-time histories of P1 and P3 of recoil spring both start from the lowest point (the lowest valley), and there is no need to rearrange the time history.

5.2.3. Fatigue Stress Spectrum Statistics. According to the counting rules and procedures of the rain flow counting method, and based on MATLAB, the rain flow counting program is compiled. The time history of the stress at the points $\mathrm{P} 1$ and $\mathrm{P} 3$ is counted to obtain the stress amplitude, the mean stress, and the number of cycles.

In the single process of multi-wire recoil spring recoil and re-entry, data statistics are performed on the dangerous point $\mathrm{P} 1$, and it is learned that a total of 102 complete stress cycles are generated. Since the statistical results of processing rain flow counts are generally expressed by the stress spectrum, the stress amplitude and mean value at point P1 are divided into 8 levels to obtain the multi-wire recoil spring fatigue stress spectrum, in which the amplitude group 


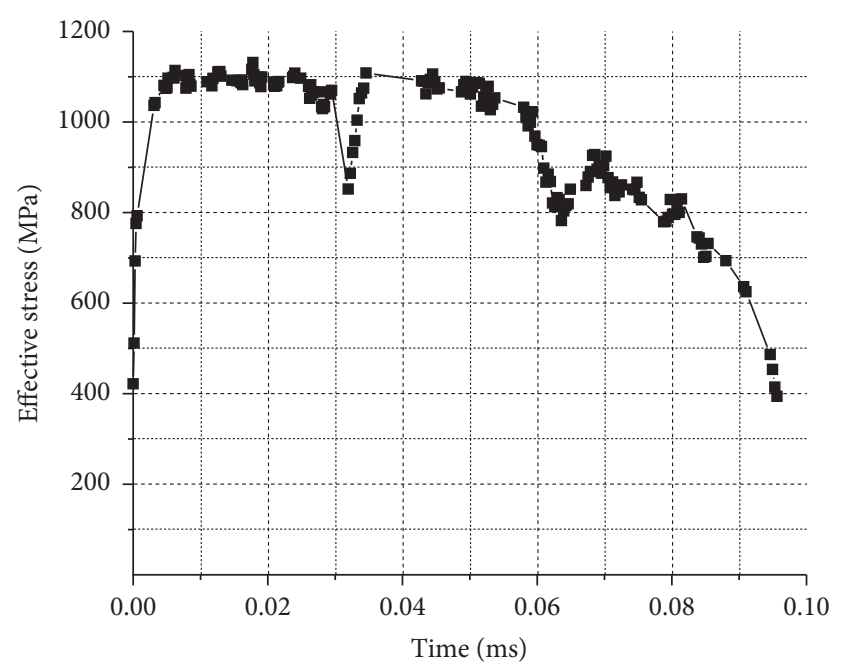

Figure 23: Effective stress-time history curve of point P1.

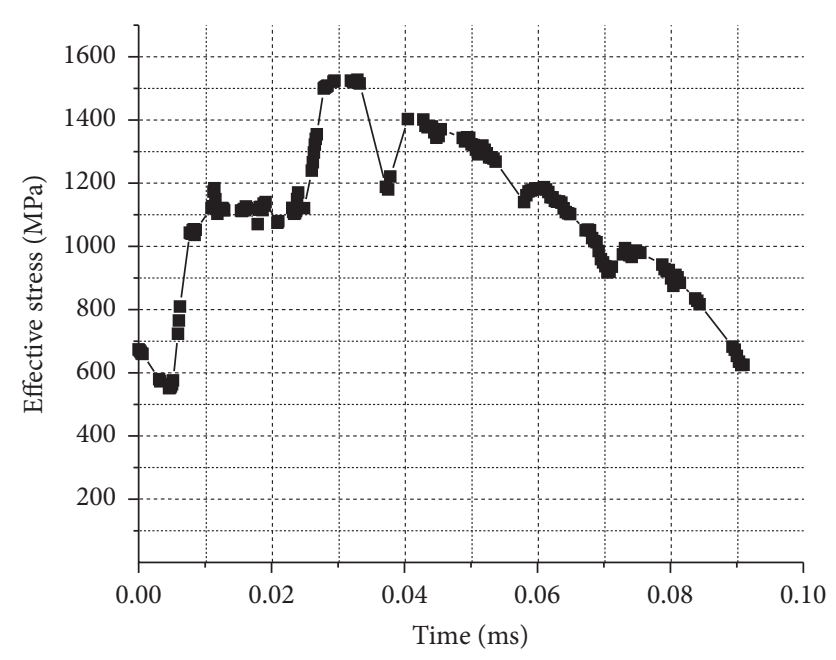

FIGURE 24: Effective stress-time history curve of point P3.

distance is 96 and the average group distance is 95 , as shown in Table 5.

Similarly, the statistics of the dangerous point P3 is obtained, and 57 complete stress cycles are obtained. The stress amplitude and average value at point $\mathrm{P} 3$ are divided into 8 levels to obtain the multi-wire recoil spring fatigue stress spectrum. The amplitude group distance is 131 and the average group distance is 130 . The stress spectrum is shown in Table 6.

5.3. Fatigue Life Prediction of Recoil Spring. By analyzing the fatigue performance of the recoil spring material, the S-N curve of the spring is obtained by modifying the S-N curve of the material, and a fatigue cumulative damage model is established to calculate the fatigue life of the recoil spring.

5.3.1. S-N Curve of Recoil Spring Material. The S-N curve of $55 \mathrm{Si} 2 \mathrm{Mn}$ generally has an exponent form, which is expressed as follows:

$$
\sigma^{m} N=C,
$$

where $m$ and $C$ are material constants.

Take the logarithm of both sides of equation (16) to get

$$
\lg N_{p}=a_{p}+b_{p} \lg \sigma .
$$

In the formula, $a_{p}$ and $b_{p}$ are stress constants. Paper [17] shows that when the survival rate of $55 \mathrm{Si} 2 \mathrm{Mn}$ is $50 \%, a_{p}=$ 34.81 and $b_{p}=-10.74$. When the survival rate is $99 \%, a_{p}=$ 29.34 and $b_{p}=-8.9$. Thus, life equations (18) and (19) and $\mathrm{S}-\mathrm{N}$ curves under different survival rates are obtained, as shown in Figure 25.

$$
\begin{aligned}
& \lg N_{50}=34.18-10.74 \lg \sigma, \\
& \lg N_{99}=29.34-8.9 \lg \sigma .
\end{aligned}
$$

5.3.2. Modified S-N Curve of 55Si2Mn. The P-S-N curves of the materials in the manual are nominal curves obtained based on standard test specimens. To obtain the multi-wire recoil spring fatigue curve, the fatigue curve of the 55Si2Mn test piece needs to be modified, and the correction coefficient is selected according to the multi-wire recoil spring operating conditions, processing technology, and load.

The multi-wire recoil spring is mostly affected by the shear stress and the friction between wire and wire. Force conversion is required, which is expressed by the load factor $C_{m}$, as shown in the following formula:

$$
C_{m}=\frac{\text { Torsional fatigue strength }}{\sigma_{s}},
$$

where $C_{m}$ of the elastoplastic material is generally taken as 0.58 .

The fatigue notch factor $K_{r}$ is used to characterize the effect of multi-wire recoil spring surface stress concentration. Its expression is

$$
K_{r}=\frac{\sigma_{s}}{\sigma_{\mathrm{sn}}}
$$

where $\sigma_{\mathrm{sn}}$ is the fatigue strength of the notch event. From the material manual, it is $466 \mathrm{MPa}$ at $P=55 \%$ and $449 \mathrm{MPa}$ at $P=99 \%$. And get $K_{r 50}=1.84$ and $K_{r 99}=2.14$.

The size factor $\varepsilon$ can characterize the effect of recoil spring size on fatigue strength, which can be expressed as

$$
\varepsilon=\frac{S_{l}}{S_{s}}=\frac{\sigma_{-1 d}}{\sigma_{-1 d 0}},
$$

where $\sigma_{-1 d}$ is the ultimate strength of the size and $\sigma_{-1 d 0}$ is the fatigue limit of the standard specimen. The diameter of the multi-wire recoil spring in this paper is $1.2 \mathrm{~mm}$, and the size factor approaches 1.

This article considers the effect of surface machining factor $\beta_{1}$ and coefficient of intensification $\beta_{2}$ on fatigue strength. $\beta_{1}$ is expressed as

$$
\beta_{1}=\frac{\sigma_{-1 a}}{\sigma_{-1}}
$$


TABLe 5: Stress spectrum at point P1 of recoil spring (unit: MPa).

\begin{tabular}{|c|c|c|c|c|c|c|c|c|}
\hline \multirow{2}{*}{ Mean stress } & \multicolumn{8}{|c|}{ Stress amplitude } \\
\hline & 47.7669 & 143.301 & 238.834 & 334.368 & 429.902 & 525.436 & 620.969 & 716.503 \\
\hline 1053.81 & 61 & 0 & 0 & 0 & 0 & 0 & 0 & 0 \\
\hline 959.218 & 10 & 0 & 0 & 0 & 0 & 0 & 0 & 0 \\
\hline 864.63 & 6 & 0 & 0 & 0 & 0 & 1 & 0 & 0 \\
\hline 770.042 & 13 & 1 & 0 & 0 & 0 & 0 & 0 & 1 \\
\hline 675.455 & 6 & 0 & 0 & 0 & 0 & 0 & 0 & 0 \\
\hline 580.867 & 1 & 0 & 0 & 0 & 0 & 0 & 0 & 0 \\
\hline 486.279 & 1 & 0 & 0 & 0 & 0 & 0 & 0 & 0 \\
\hline \multirow[t]{2}{*}{391.691} & 1 & 0 & 0 & 0 & 0 & 0 & 0 & 0 \\
\hline & & & & Cycles & & & & \\
\hline
\end{tabular}

TABLE 6: Stress spectrum at point P3 of recoil spring (unit: MPa).

\begin{tabular}{|c|c|c|c|c|c|c|c|c|}
\hline \multirow{2}{*}{ Mean stress } & \multicolumn{8}{|c|}{ Stress amplitude } \\
\hline & 65.6152 & 196.846 & 328.076 & 459.307 & 590.537 & 721.767 & 852.998 & 984.228 \\
\hline 1525.64 & 2 & 0 & 0 & 0 & 0 & 0 & 0 & 0 \\
\hline 1395.71 & 5 & 0 & 0 & 0 & 0 & 0 & 0 & 0 \\
\hline 1265.78 & 2 & 0 & 1 & 0 & 0 & 0 & 0 & 0 \\
\hline 1135.85 & 23 & 0 & 0 & 0 & 0 & 0 & 0 & 1 \\
\hline 1005.92 & 8 & 0 & 0 & 0 & 0 & 0 & 0 & 0 \\
\hline 875.989 & 5 & 0 & 0 & 0 & 0 & 0 & 0 & 0 \\
\hline 746.058 & 2 & 0 & 0 & 0 & 0 & 0 & 0 & 0 \\
\hline 616.127 & 4 & 0 & 0 & 0 & 0 & 0 & 0 & 0 \\
\hline & & & & Cycles & & & & \\
\hline
\end{tabular}

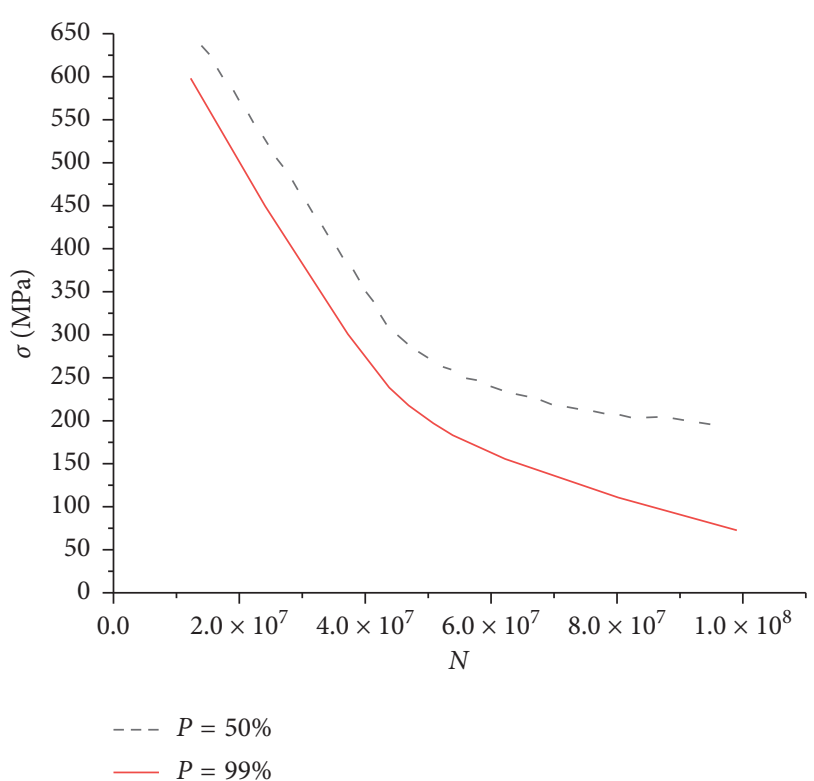

FIGURE 25: S-N curve of multi-wire recoil spring.

where $\sigma_{-1 a}$ is the fatigue limit of a standard smooth specimen with a certain processed surface, and select $\beta_{1}=0.7$ according to the surface machining factor curve graph.

The surface of the multi-wire recoil spring needs to be strengthened, which is characterized by the coefficient of intensification $\beta_{2}$, and its expression is as follows:

$$
\beta_{2}=\frac{\sigma_{-1 q}}{\sigma_{-1}}
$$

where $\sigma_{-1 q}$ is the bending fatigue limit of the specimen after the strengthening treatment. The yield limit of $55 \mathrm{Si} 2 \mathrm{Mn}$ after the hardening treatment of $880^{\circ}$ oil reaches $1715 \mathrm{Mpa}$, and we have $\beta_{2}=1.75$.

Combining the above effects, the correction factor $K_{\alpha}$ is obtained:

$$
K_{\alpha}=f\left(K_{r}, \mathcal{\varepsilon}, \beta_{1}, \beta_{2}, C_{m}\right)=\frac{\left(\left(K_{r} / \varepsilon\right)+\left(1 / \beta_{1} \beta_{2}\right)-1\right)}{C_{m}},
$$

$K_{\alpha 50}=2.06$ and $K_{\alpha 99}=2.26$ are calculated from equation (25).

5.3.3. Fatigue Life Calculation. According to the S-N curve and statistical stress spectrum of the multi-wire recoil spring obtained above, and based on the Miner theory, the cumulative fatigue damage within one stroke of recoil spring is obtained as

$$
D_{L}=\sum_{i=1}^{k} \frac{n_{i}}{N_{i}}
$$

where $k$ is the level of stress level, $n_{i}$ is the number of times that the $i$-th level stress cycle occurs in the stress spectrum, and $N_{i}$ is the number of failure cycles under the $i$-th load alone, which is obtained from the S-N curve.

Calculate the cumulative fatigue damage according to the above formula and the $\mathrm{S}-\mathrm{N}$ curve of recoil spring. And the cumulative damage and number of strokes of the multiwire recoil spring in a stroke are shown in Table 7. 
TABle 7: Cumulative damage and fatigue life of multi-wire recoil spring.

\begin{tabular}{lcccc}
\hline \multirow{2}{*}{ Properties } & \multicolumn{2}{c}{ Reliability $P=50 \%$} & \multicolumn{2}{c}{ Reliability $P=99 \%$} \\
& $P 1$ & $P 3$ & $P 1$ & $P 3$ \\
\hline $\begin{array}{l}\text { Cumulative damage } \\
\text { in one stroke }\end{array}$ & $5.4 e-5$ & $4.9 e-5$ & $7.8 e-4$ & $7.5 e-4$ \\
Number of strokes & 18273 & 20408 & 1282 & 1333 \\
\hline
\end{tabular}

The number of strokes of the recoil spring predicted by the fatigue life curve when the reliability $P=50 \%$ is 18273 . And combining the comprehensive life of the gun [18] and the general experimental results, the life of the recoil spring is generally around 20,000 strokes. The calculation result is $8.6 \%$ different from the experiment value, which proves that the method has certain reliability. In addition, from Table 7 and the above calculations, it can be seen that the correction factor plays an extremely critical role in the prediction of fatigue life. To ensure accurate prediction of recoil spring fatigue life, it is necessary to accumulate experiments and relevant experience on the processing of correction coefficients while determining the accurate load spectrum.

\section{Conclusions}

This paper studies the impact characteristics and fatigue life of the multi-wire recoil spring. Firstly, we carry out a parametric modeling of the recoil spring. Secondly, the dynamic response characteristics of the recoil spring under the impact are analyzed by finite element method, and experimental verification is performed. Then, based on the stress-time history curve of the dangerous position obtained by the finite element analysis, the statistical calculation is performed by the rain flow counting method to obtain the fatigue stress spectrum of recoil spring. Finally, according to the Miner damage theory, the fatigue life prediction of the recoil spring material based on the improved S-N curve of the spring material is made, and compare the calculation results with experimental values. The following conclusions are reached:

(1) During the impact of multi-wire recoil spring, a stress wave propagating to the fixed end of the spring is generated in the coil, and the maximum stress value is reached at the fixed end.

(2) When the impact velocity is greater than the stress wave propagation velocity, the coils will easily be combined during the impact process, and the maximum stress of the multi-wire recoil spring will not occur on the inside of the coil not like the ordinary single-wire cylindrically helical spring, but at the position where the wire and wire is in contact.

(3) The fatigue life of the multi-wire recoil spring obtained under a continuous impact load using a fatigue curve with reliability $P=50 \%$ is in good agreement with the experimental results. And the calculation results show that the correction factor plays an extremely critical role in the prediction of fatigue life. To ensure accurate prediction of recoil spring fatigue life, it is necessary to accumulate experiments and relevant experience on the processing of correction coefficients while determining the accurate load spectrum. And it also shows that the fatigue life of the multi-wire recoil spring predicted by the modified material S-N curve has certain reference value for its design.

\section{Data Availability}

Relevant research data can be obtained upon request to the corresponding author.

\section{Conflicts of Interest}

The authors declare that they have no conflicts of interest.

\section{Acknowledgments}

This work was supported by the National Defense Basic Scientific Research Project under Grant A1020131011.

\section{References}

[1] Y. Peng, S. Wang, J. Zhou, and S. Lei, "Structural design, numerical simulation and control system of a machine tool for stranded wire helical springs," Journal of Manufacturing Systems, vol. 31, no. 1, pp. 34-41, 2012.

[2] J. W. Phillips and G. A. Costello, "General axial response of stranded wire helical springs," International Journal of Nonlinear Mechanics, vol. 14, no. 4, pp. 247-257, 1979.

[3] G. A. Costello and J. W. Phillips, "Static response of stranded wire helical springs," International Journal of Mechanical Sciences, vol. 21, no. 3, pp. 171-178, 1979.

[4] J. Lee, "Free vibration analysis of non-cylindrical helical springs by the pseudospectral method," Journal of Sound and Vibration, vol. 305, no. 3, pp. 543-551, 2007.

[5] D. Yu, "Vibration and dynamic stress of recoil spring coil," Journal of China Ordnance, vol. 1, no. 1, pp. 1-12, 1980.

[6] J. Min and S. Wang, "Analysis on dynamic calculation of stranded wire helical spring," Chinese Journal of Mechanical Engineering, vol. 43, no. 3, pp. 199-203, 2007.

[7] S. Wang, S. Lei, J. Zhou et al., "Research on the impact response of the two ends of the spring," Journal of Vibration and Shock, vol. 30, no. 3, pp. 64-68, 2011.

[8] S. Wang, B. Tian, Y. Zhao et al., "Improved shock load model of stranded wires helical springs based on perturbation method," Journal of Mechanical Engineering, vol. 51, no. 7, pp. 85-90, 2015.

[9] D. Čakmak, Z. Tomičević, H. Wolf et al., "Vibration fatigue study of the helical spring in the base-excited inerter-based isolation system," Engineering Failure Analysis, vol. 103, pp. 44-56, 2019.

[10] M. Zhang, "The analysis of spring's fatigue life based on eternal fatigue life combined with S-N curve[J]," Mechanical Research \& Application, vol. 10, no. 6, pp. 107-113, 2012.

[11] D. Yu, X. Li, C. Wang et al., "Reliability design of the fatigue strengthen of cylindrical spiral spring," Machinery Design \& Manufacture, vol. 8, no. 8, pp. 19-20, 2007.

[12] S. Lei, Research on Impact Characteristic and Damage Mechanism of Stranded-Wire Helical Spring, Chongqing University, London, UK, 2010. 
[13] A. N. Chaudhury and D. Datta, "Analysis of prismatic springs of non-circular coil shape and non-prismatic springs of circular coil shape by analytical and finite element methods," Journal of Computational Design and Engineering, vol. 4, no. 3, pp. 178-191, 2017.

[14] D. Fakhreddine, A. T. Mohamed, and H. D. MohamedAbderrazek, "Finite element method for the stress analysis of isotropic cylindrical helical spring," European Journal of Mechanics-A/Solids, vol. 24, no. 6, pp. 10681078, 2005.

[15] A. Khosrovaneh and N. Dowling, "Fatigue loading history reconstruction based on the rainflow technique," International Journal of Fatigue, vol. 12, no. 2, pp. 99-106, 1990.

[16] C. Yan and G. Wang, "Study on the rain flow counting method and its statistical processing program," Journal of Agricultural Machinery, vol. 12, no. 4, pp. 90-103, 1982.

[17] Z. Zeng, Handbook of Mechanical Engineering materials, Mechanical Industry Press, Beijing, China, 2009.

[18] Y. Qi and C. Xu, "Study on the wear and performance degradation of large caliber machine gun during the lifetime of the whole projectile," Acta Armamentarii, vol. 37, no. 8, pp. 1359-1364, 2016 